Alexander Z̈̈H

\title{
ZUR LOKALISIERUNG VON KARYANDA IN KARIEN
}

Die Lage der karischen Polis Karyanda, die von diversen antiken Autoren genannt und im 5. Jahrhundert v. Chr. als Mitglied des Delisch-Attischen Seebundes in den athenischen Tributlisten erwähnt wird, ist bis heute noch nicht mit Sicherheit bestimmt worden ${ }^{1}$. Im Zuge der Feldforschung zur Dissertation »Zur Typologie kirchlicher Architektur im südwestlichen Kleinasien« ist der Verfasser auf eine bisher unbekannte antike Ortslage bei Güvercinlik (ca. 20 km nordöstlich von Halikarnassos/Bodrum) aufmerksam geworden, die es erlaubt, der begonnenen Diskussion einen weiteren Vorschlag hinzuzufügen. Bereits Wolfgang Radt hat darauf hingewiesen, daß die Erforschung der Halbinsel von Halikarnassos und ihrer Umgebung bei weitem noch nicht abgeschlossen ist und hier noch ungezählte antike Stätten und Siedlungen einer weiteren wissenschaftlichen Erschließung harren².

\section{Antike Überlieferung ${ }^{3}$}

Eine Hauptquelle für die Lokalisierung von Karyanda ist die Beschreibung und Erwähnung im Text des Skylax von Karyanda ${ }^{4}$ - einer Seeroutenbeschreibung, die offenbar in hellenistischer

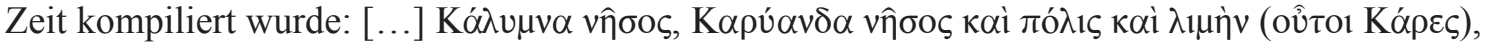

\footnotetext{
Die Abkürzungen folgen den ÖJh 69, 2000, 357 ff. Zusätzlich werden verwendet:

Bean - Cook, Peninsula J. E. Bean - J. M. Cook, The Halicarnassus Peninsula, BSA 50, 1955, 85-171.

Foss, Strobilos

C. Foss, Strobilos and Related Sites, AnatSt 38, 1988, 147-174.

McNicoll, Fortifications

A. W. McNicoll, Hellenistic Fortifications from the Aegean to the Euphrates (1997).

Meritt - Wade-Gery - McGregor, B. D. Meritt - H. T. Wade-Gery - M. F. McGregor (Hrsg.), The Athenian Tribute Tribute Lists

Newton, History

Lists I (1939).

Ch. T. Newton, A History of Discoveries at Halicarnassus, Cnidus and Branchidae [...] I-II und Tafelband (1861-63).

Newton, Travels

Paton - Myres, Sites

Ch. T. Newton, Travels and Discoveries in the Levant I-II (1865)

W. R. Paton - J. L. Myres, Karian Sites and Inscriptions, JHS 16, 1896, 188-236 (Part I); 237-271 (Part II) Taf. 9-11.

Paton - Myres, Karyanda

W. R. Paton - J. L. Myres, Three Karian Sites: Telmissos, Karyanda, Taramptos, JHS 14, 1894, 373-377.

Radt, Siedlungen

W. Radt, Siedlungen und Bauten auf der Halbinsel von Halikarnassos, 3. Beih. IstMitt (1970).

Ruggieri - Giordano - Zäh, $\quad$ V. Ruggieri - F. Giordano - A. Zäh, La penisola di Alicarnasso in età bizantina. Peninsola

I. Parte, Orientalia Christiana Periodica 63, 1997, 119-161.

1 Bean - Cook, Peninsula 155: »The site of Caryanda is a long-standing enigma.« Vgl. RE X (1919) 2246-2247 s. v. Karyanda (Bürchner); R. Stillwell (Hrsg.), The Princeton Encyclopedia of Classical Sites (1976) 798 s. v. Salihadas1 (G. E. Bean); Der Neue Pauly VI (1999) 310 s. v. Karyanda (H. Kaletsch). Zum Delisch-Attischen Seebund: H. Bengtson, Griechische Geschichte ${ }^{9}$ (2002) 161 ff. (= HAW III $4^{5}$ [1977] ohne wiss. Apparat). Inschriftliche Belege der Polis in den Listen mit Jahresangaben bei: Meritt - Wade-Gery - McGregor, Tribute Lists 300301.

2 Radt, Siedlungen 15-16.

3 Diskussion aller relevanten Textpassagen mit unterschiedlichen Ergebnissen: Newton, History II 598 f.; Meritt Wade-Gery - McGregor, Tribute Lists 498; Bean - Cook, Peninsula 155 ff.

${ }^{4}$ Der Kleine Pauly V (1979) 238 s. v. Skylax von Karyanda (F. Lasserre).
} 


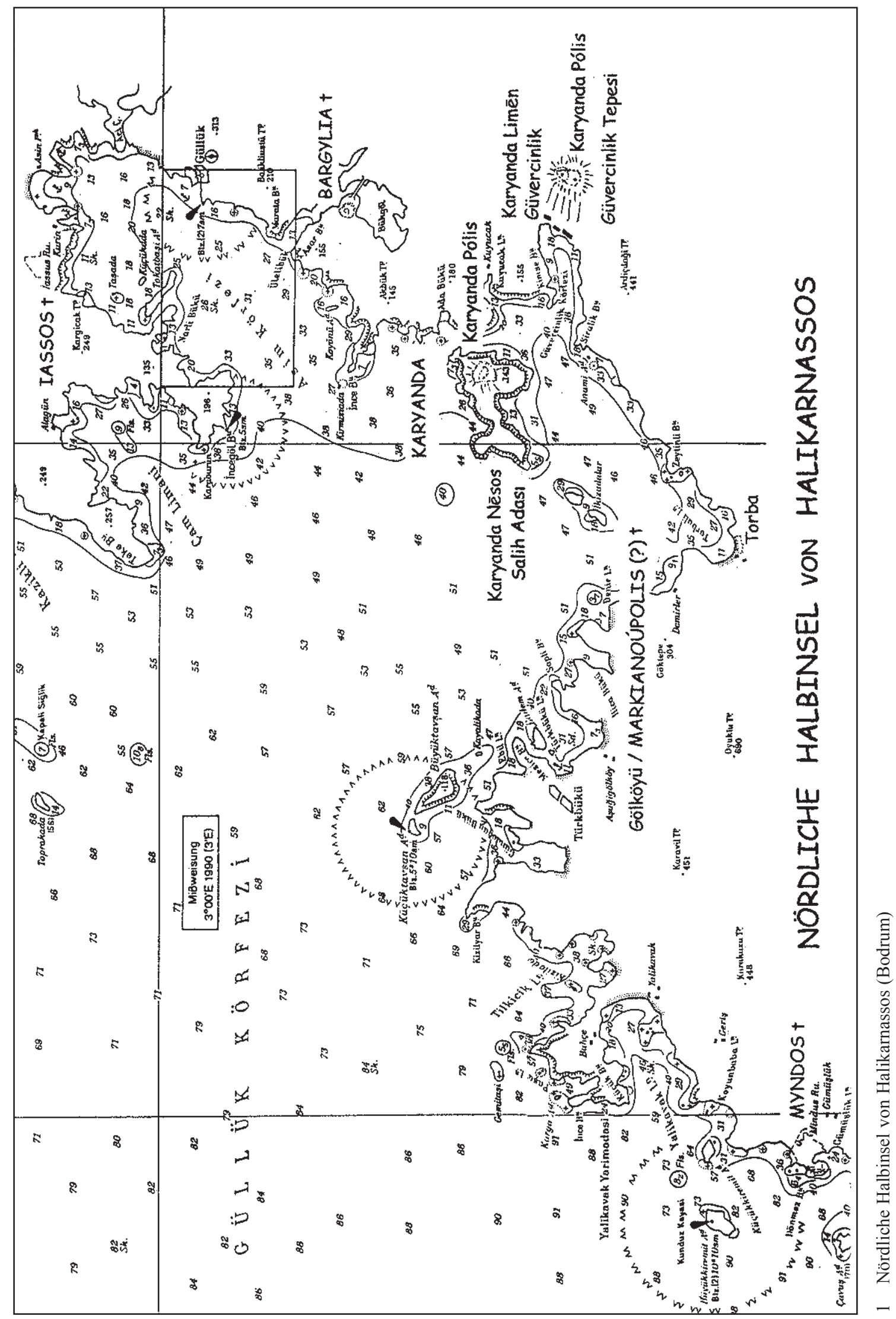




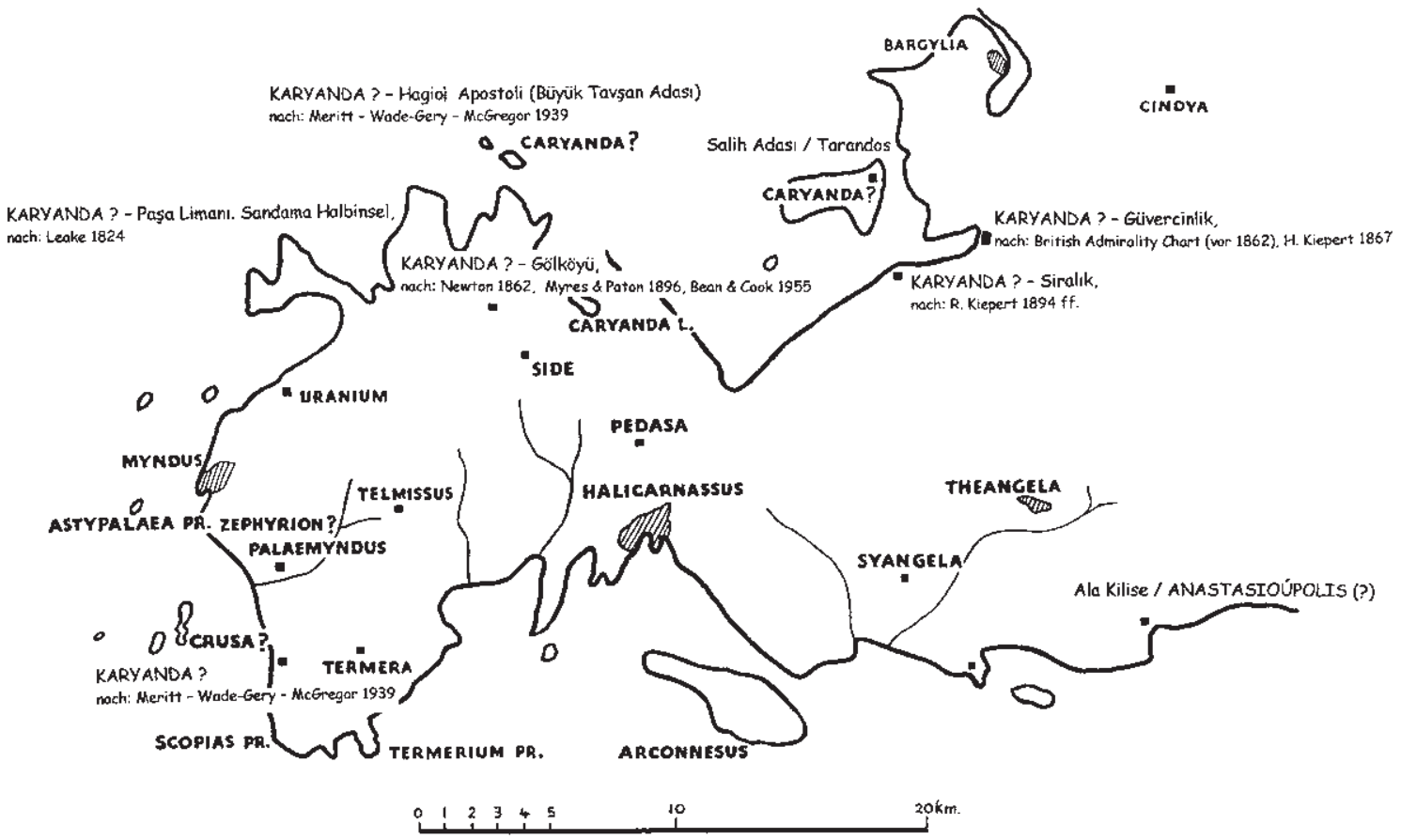

2 Übersichtskarte zur historischen Lokalisierungsdiskussion

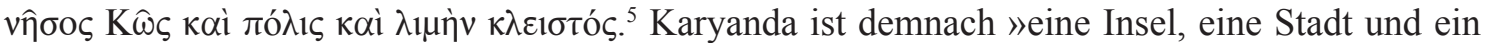
Hafen« in Karien, aufgezählt mit den Inseln Kalymnos und Kos. Durch diese Aufzählung ist zunächst nicht klar, ob - ähnlich der gleich folgenden Nennung der Insel Kos - hiermit nicht etwa nur eine einzige Stätte beschrieben und gemeint sein könnte.

Im Folgenden wird klarer, daß Karyanda offenbar eine Doppelidentität hatte. Zunächst wird Karyanda - nach Caius Plinius Secundus - präziser als Stadt und nicht explizit als Insel zwischen den heute unstrittig identifizierten Poleïs von Myndos $^{6}$ und Bargylia ${ }^{7}$ angegeben (vgl. Abb. 1): »Weiterhin folgen Myndos und (die Stelle) wo Alt-Myndos (Palaemyndos) lag, Nariandos [Durvanda - Torba?], Neapolis [Gölköyü?], Karyanda, Termera, das freie Bargylia und die Stadt Iasos [...]. $\ll^{8}$ Nach Plinius und Strabon von Amaseia müssen wir davon ausgehen, daß es - neben der Insel - auch noch eine festländische Siedlung Karyanda gegeben hat. »[...] dann sofort Myndos mit einem Hafen, nach dieser Bargylia, gleichfalls eine Stadt; zwischen beiden der Hafen Karyanda und eine ihm gleichnamige Insel, welche die Karyander bewohnten. Hier war auch Skylax gebürtig $[\ldots] . \ll^{9}$

5 Scylacis Caryandensis, Periplus §99, in: C. Müller (Hrsg.), Geographi Graeci Minores I-III (1855, Nachdruck 1990) I 72-73.

6 McNicoll, Fortifications 22-23.

7 Zuletzt: A. Zäh, Die Stiftungsinschrift der oberen Basilika von Bargylia, EpigrAnat 34, 2002, 117-118.

8 Plin. nat. 5, 29, 107 (Ed./Übersetzung R. König [1993] 78-81). Vgl. dazu: Bean - Cook, Peninsula 156-157; zu Myndos: ebenda 145; Termera: ebenda 147 ff.; Durvanda = Torba: ebenda 159.

9 Strab. 14, 2, 20. Übersetzungen: Ch. G. Grosskurd, Strabon's Erdbeschreibung in Siebzehn Büchern, III. Theil (1833) 53-54. A. Forbiger, Strabo III., Buch 11-15 (1858) 118-119. Gefolgt wird dieser Darstellung im Ortsnamenslexikon des Stephanos von Byzanz (5. Jh.) durch folgende Kurzmitteilung, die allerdings wieder ungenauere Informationen zu bieten scheint: »Karyanda - Stadt und gleichnamiger Hafen in der Nähe von Myndos und Kōs.» Stephanus von Byzanz, s. v. Karyanda. Editionen: A. Westermann (1839); A. Meineke (1849, Nachdruck 1958). Zur Wahrnehmung der Geographie und der tatsächlichen Stichhaltigkeit der geographischen Literatur in der Antike vgl. die grundlegende Studie von K. Brodersen, Terra Cognita. Studien zur Römischen Raumerfassung², Spudasmata 59 (2003). 


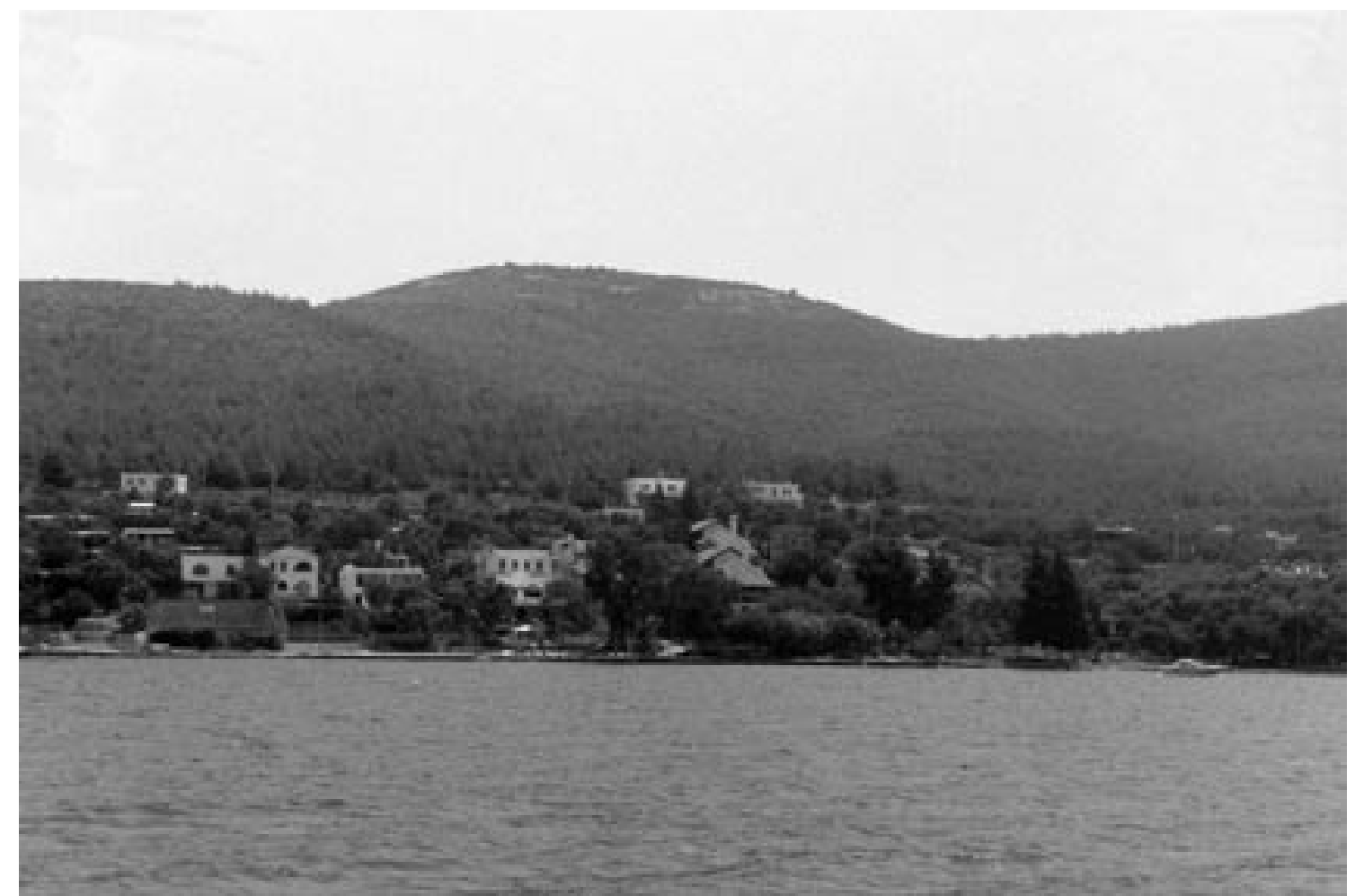

3 Güvercinlik Tepesi, von der Güvercinlik Bucht aus aufgenommen

Weitere und genauere Angaben zur Lokalisierung sind den antiken Schriftquellen nicht zu entnehmen. Andere historische Notizen erlauben es, die Bedeutung der Siedlung anhand der niedrigen Abgaben ( $\phi o ́ p o l$ ) als gering zu relativieren ${ }^{10}$.

\section{Lokalisierungsdiskussion}

Die Diskussion der Ortslage auf Basis der schriftlichen Überlieferung setzt mit dem Aufkommen der historischen Kartographie im ausgehenden 18. Jahrhundert und den ersten Forschungsreisenden in der ersten Hälfte des 19. Jahrhunderts ein (Abb. 2). Auf zahlreichen Karten dieser Epoche ist der Ortsname als Insel und Hafen zwischen den zu dieser Zeit schon verbindlich lokalisierten Poleïs von Myndos und Bargylia eingetragen ${ }^{11}$. Einer der frühesten präziseren Lokalisierungsvorschläge stammt von dem berühmten britischen Geographen Colonel William Martin Leake, der Karyanda (Paşa Limanı) hypothetisch auf der Sandama-Landzunge (heute: Yalıkavak-Halbinsel) der nordwestlichen Halbinsel von Halikarnassos (Bodrum) vermutet, ohne daß dies jedoch durch archäologische Befunde zu bestätigen gewesen wäre ${ }^{12}$. Erstmals bei Güvercinlik scheint

10 Bean - Cook, Peninsula 156. 159. Zu den Abgaben vgl. auch RE X (1919) 2246-2247 s. v. Karyanda (Bürchner).

11 z. B. J. B. B. d'Anville, Atlas Antiquus Anvillianus (1784) B1. 7: Asia Minor et Syria »Caryanda«; P. Lapie, Carte Générale de la Turquie d'Europe en XV Feuilles (1822): vgl. hier die B1. 12: »Caryanda I.[le]« und XV »Port

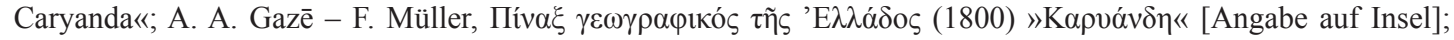
K.[u.]K. Öster. Generalquartiermeisterstab (Hrsg.), F. von Weiss, Carte der Europäischen Türkey nebst einem Theile von Kleinasien in XXI Blättern (1829): vgl. hier Bl. 18: Rodos »I.[nsel] Kavergina (I. Carianda)«.

12 W. M. Leake, Journal of a Tour in Asia Minor [...] (1824, Nachdruck 1976) 227-229, vgl. ebenda die Karte; ders., Essay of a Map of Asia Minor (1822); Myres - Paton, Sites $261 \mathrm{f}$. Taf. $11 »$ Map of the Peninsula of Myndos (Halikarnassos) from Surveys by Messrs. Myres and Paton« (nur Felsengräber); Bean - Cook, Peninsula $130 » \ldots$ scattered traces of late classical and christian occupation along the bay of Sandıma and Yalıkavak ...«. Gerade die 
Karyanda auf französischen und österreichischen Karten $^{13}$ und der Seekarte der Britischen Admiralität angesetzt zu sein, ohne es jedoch archäologisch nachzuwei$\operatorname{sen}^{14} .1867$ folgt dem auch der deutsche Geograph Heinrich Kiepert in seinem Kartenwerk »Atlas Antiquus « und lokalisiert die Stadt Karyanda bei Güvercinlik, sein Sohn Richard später auf einer winzigen Landzunge (Siralık) ein wenig westlich von Güvercin-

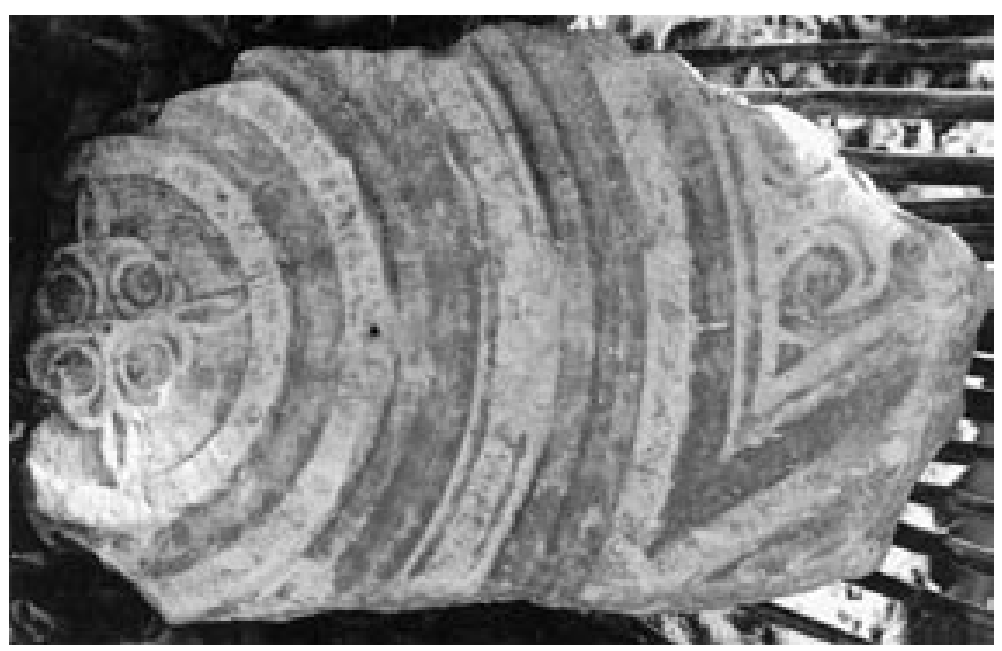

4 Güvercinlik, Teehaus. Fragment einer byzantinischen Reliefplatte

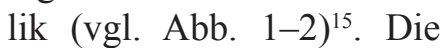

Ruinen dort sind aber sehr gering und eignen sich kaum, diese Siedlung als kleine Polis zu interpretieren ${ }^{16}$.

Seit dem 19. Jahrhundert wird die heutige türkische Salih Adas1 (neugriech.: Tarandos- oder Taranta-Insel) ${ }^{17}$, die größte Insel, die an der Küste zwischen Myndos und Bargylia liegt und auf der man die Inselsiedlung von Karyanda vermutet, für Karyanda gehalten. Dafür gibt es stichhaltige archäologische Argumente. Dazu zählen Inschriften aus antiker und frühchristlicher $Z_{\text {Zeit }}{ }^{18}$, die möglicherweise auf der Insel gefunden wurden und wahrscheinlich auch von der Insel stammen; sie legen hier eine bedeutendere Siedlung nahe. Hinzu treten noch ausgedehnte frühe, möglicherweise lelegische Ruinen auf den Anhöhen im nordöstlichen Teil der Insel ${ }^{19}$.

Weitergeführt wurde die Diskussion über die Ansetzung der festländischen Polis Karyanda von den Briten W. R. Paton und J. L. Myres, die in den 90er Jahren des 19. Jahrhunderts die Halbinsel von Halikarnassos intensiv erforschten und Karyanda - wie Ch. T. Newton 1862 - auf-

von A. Philippson an dieser Stelle attestierte »Küstensenkung« (vgl. u. Anm. 22) macht Leakes Hypothese, nach der die Sandama-Halbinsel in der Antike eine Insel gewesen sei, auch geographisch unglaubwürdig.

13 Vgl. o. Anm. 11.

14 Newton, History II 600: »In the Admirality Chart [No. 1546], and also in the map of Caria in the work of Lebas [= M. Ph. Le Bas, Voyage archéologique en Grèce et en Asie Mineure fait par Ordre du Governement Français pendant les Années 1843 et 1844. Itinéraire I $2\left(1847,1856^{2}\right)$ ], Caryanda is placed at Guverjilik, at the head of the gulf, Tarandos being thus identified with the island mentioned by Scylax. But at Guverjilik there is neither lake nor ancient remains [!?], ...«. Paton - Myres, Karyanda 375 erwähnen zwar Güvercinlik als »the deep bay with ruins and traces of a road«, verwerfen diese Möglichkeit aber sofort.

15 H. Kiepert, Atlas Antiquus. Zehn Karten zur Alten Geschichte ${ }^{4}$ (1867) B1. 8: »Inseln und Asiatische Westküsten. Maßstab 1:1.000.000«; Paton - Myres, Sites, Taf. 10, Map of Part of Karia »Karyanda (Kiepert)«; R. Kiepert, Karte von Kleinasien in 24 Blättern² (1911) Bl. D1 »Budrúm«.

16 Foss, Strobilos 169 f. Taf. 18a-b; Bean - Cook, Peninsula $157 \mathrm{f}$.

17 Der neugriechische Name der Insel ist nicht mit dem für diese Gegend belegten, aber völlig unbestimmten antiken Ortsnamen »Taramptos« gleichzusetzten. Vgl. Bean - Cook, Peninsula 159. So noch: Meritt - Wade-Gery - McGregor, Tribute Lists 498. Paton - Myres, Karyanda 375.

18 H. Grégoire (Hrsg.), Recueil des Inscriptions Grècques Chrétiennes d'Asie Mineure (1922) 78 No. 230 [= Waddington - Le Bas No. 500, wie folgt]; W. H. Waddington - M. Ph. Les Bas, Voyage Archéologique en Grèce et en Asie Mineure, fuit pendant les années 1834 et 1844 (1847-77) II, 4-5; Inscriptions Grècques et Latines en Asie Mineure (1870, Nachdruck 1972) Section II. Carie - VII. »Karyanda«, 158 Nos. 499. 500; B. Haussoullier, Inscription de l'Île de Karyanda, BCH 8, 1884, 218-222. Die Herkunftsangaben der Inschriften sind in den Inschriften-Korpuswerken allerdings oft nicht korrekt und ungenau. Richtigstellungen zu Grégoire (a. O.) bereits: L. Robert, Villes d'Asie Mineure² (1962) 148 (Anm. 1).

19 Bean - Cook, Peninsula 132. Autopsie durch den Verf. 1996. 


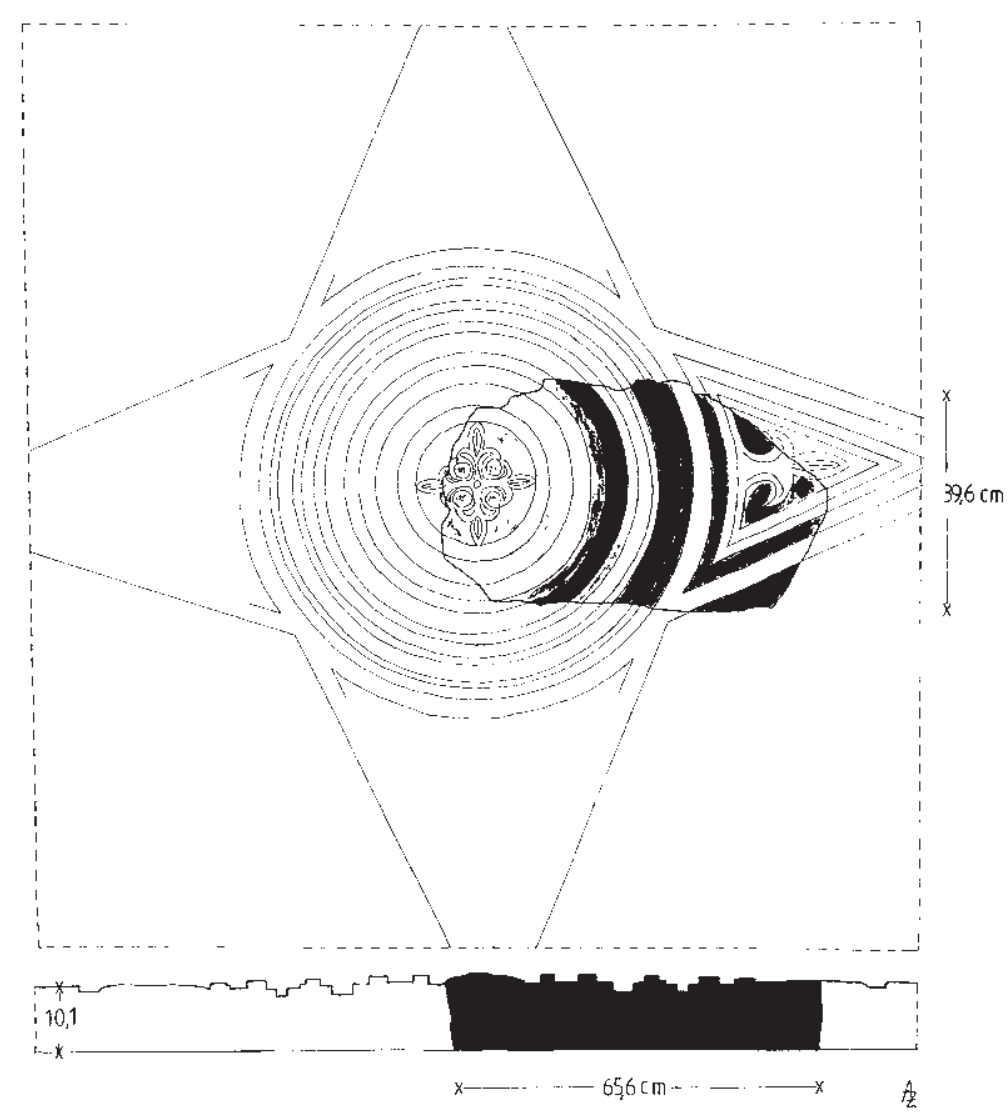

5 Byzantinische Reliefplatte, Wiederherstellungsversuch grund eines neuen einzigen 'theorieerhärtenden' Münzfundes beim heutigen Gölköyü auf der nördlichen Halbinsel von Halikarnassos anzusetzen gedachten ${ }^{20}$. Eine Unsicherheit in der Textüberlieferung von Strabon und Stephanos, die Karyanda auch als einen »See« (griech.: $\gg \lambda i \mu \nu \eta \eta \ll, \quad$ türk.: »göl« im Unterschied zu $» \lambda ı \mu n v \ll-»$ Hafen «) erscheinen lassen kann, wird hier erstmals von dem Briten Ch. T. Newton, gefolgt von J. E. Bean und R. M. Cook, auf einen Sumpf östlich von Gölköyü bezogen ${ }^{21}$. Dies allerdings, ohne zu berücksichtigen, daß der Sumpf in der Antike sehr wahrscheinlich gar kein See gewesen war, sondern aufgrund der geographisch attestierten »Senkung der Küste seit dem Altertum $\ll^{22}$ (d. h. An-

stieg des Meeresspiegels und mögliche Grundwasseranhebung) erst in jüngerer Zeit entstanden sein mag. Ein See, der im Altertum existierte, kann im ganzen Gebiet nicht nachgewiesen werden. Allerdings gibt es auf einer Anhöhe südlich von Gölköyü ein größeres lelegisches Ruinengebiet, welches schon von W. R. Paton und J. L. Myres für Karyanda gehalten wurde. J. E. Bean und R. M. Cook denken an eine spätere Übersiedlung der Bewohner von Karyanda (Salih Adas1) nach dieser Stätte bei Gölköyü (Karyanda-Neapolis) - diese Hypothese erscheint grotesk ${ }^{23}$. Die hiermit aufgestellte Theorie, daß ein auf der Insel gelegenes 'Alt-Karyanda' früher existierte, ist obsolet $^{24}$. Aufgrund der byzantinischen Ruinen von Gölköyü, das selbst direkt am Meeresufer

20 Paton - Myres, Karyanda 375 f. Basierend auf: Newton, History II 598 f.

21 Newton, History II 598 f.; Bean - Cook, Peninsula 155 f. Zuletzt: Foss, Strobilos 172: »There is little doubt that Göl or Gölör occupies the site of ancient Caryanda ...«. In einigen überlieferten Handschriften von Strabon und Stephanos von Byzanz begegnet das offenbar zu »See = limnē« verschriebene »limēn«, das Newton sowie Bean - Cook für authentisch halten. Dem steht die zweifelsfreie Überlieferung von »limēn« des Pseudo-Skylax entgegen; s. o. Anm. 5.

22 A. Philippson, Reisen und Forschungen im westlichen Kleinasien (V. Heft), 183. Ergh. Petermann's Geographische Mitteilungen (1915) 51. Philippson attestiert dies aufgrund von Ausarbeitungen antiker Steinbrüche an der Sandama-Halbinsel (vgl. Abb. 1-2), die gerade unter der Wasseroberfläche zu erkennen waren. Zur geographischen Bedeutung solcher Beobachtungen vgl. bes. H. Gierloff-Emden, Anstieg des Meeresspiegels in historischer Zeit und versunkene Bauwerke (Archäologische Befunde), in: ders., Lehrbuch der Allgemeinen Geographie V 2. Geographie des Meeres - Ozeane und Küsten (1979) 941 ff. 1229. W. Koehne, Grundwasserkunde² (1948) 108 ff.

23 Bean - Cook, Peninsula 121 f. Abb. 9; 157 mit Anm. 302. Vgl. Paton - Myres, Sites, Taf. 11, Map - südlich von »Ghiul [Göl] Bay«; Radt, Siedlungen, Beil. 11 (Karte der Halbinsel von Halikarnassos). W. Radt behandelt den Ort nicht, er ist nur in der Karte als »Kaleyıkığı Tepe« eingetragen.

${ }^{24}$ Der Neue Pauly VI folgt hier unkritisch Bean - Cook, Peninsula sowie der Princeton Encyclopedia of Classical Sites (Bean), gemäß dem zunächst die Siedlung Karyanda auf der Salih-Insel existiert habe; vgl. o. Anm. 1. 
liegt, so auch eines Baptisteriums, können hier eine spätantike Stadt und ein Bistum vermutet werden, das möglicherweise mit Markianoupolis gleichzusetzen is $\mathrm{t}^{25}$.

Ende der 30er Jahre des 20. Jahrhunderts verwerfen die Herausgeber der athenischen Tributlisten die bis dahin vorgelegten Lokaliserungsvorschläge und möchten die Insel Karyanda südlich von Myndos auf einer der kleineren vorgelagerten Inseln vermuten, ohne dies archäologisch belegen $\mathrm{zu}$ können ${ }^{26}$. Sie fordern zudem die Überprüfung der archäologischen Fakten der zweitgrößten Insel zwischen Myndos und Bargylia, nämlich von Hagioi Apostoloi (heute: Büyük Tavşan Adası - Große Haseninsel), welche inzwischen erfolgt ist: Die Insel hat geringe hellenistische Spuren, der Verfasser stellte dort 1996 in Autopsie den Rest einer hellenistischen Mauer fest. Auf der Bergkuppe wurde - oberhalb des antiken Mauerzugs - in der Spätantike eine ton-

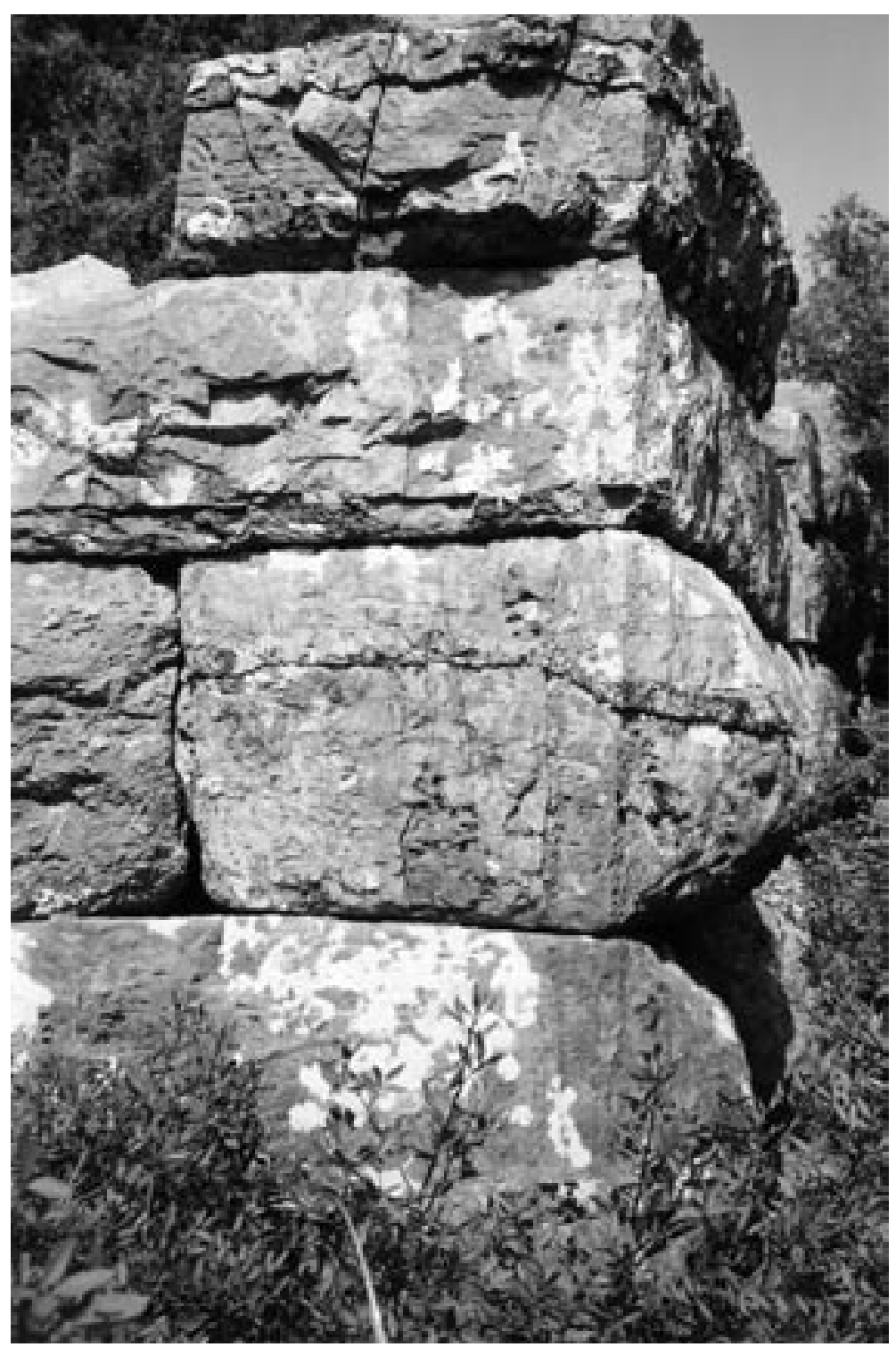

6 Güvercinlik Tepesi. Mauerwerk des hellenistischen Turms, Nordostecke nengewölbte dreischiffige

Klosterbasilika mit einer großen Zisterne errichtet. Eine Siedlung, die den Charakter einer Polis gehabt haben könnte, existierte auf dem Eiland jedoch nie ${ }^{27}$.

25 Ruggieri - Giordano - Zäh, Penisola 126 mit Anm. 9; 153 ff. Abb. 11. 43-48; Foss, Strobilos 172 f. mit Anm. 98 Taf. 18c. Ein anderes bisher nicht identifiziertes Bistum in Karien liegt östlich von Halikarnassos (Bodrum), vgl. dazu: A. Zäh, Anastasioúpolis? Der Ruinenort in der Bucht von Ala Kilise in Karien - Eine spätantike Hafenstadt, AW 34, 2003, 43-48.

26 Bean - Cook, Peninsula $158 \mathrm{f}$.

27 Die Insel wird in älteren Publikationen und Karten meist als »Hag. Apostoli« angesprochen. Autopsie durch den Verf. 1996. Bean - Cook, Peninsula 131. 159. - Zum Kloster: V. Ruggieri - F. Giordano, La penisola di Alicarnasso in età bizantina. II. Parte. Orientalia Christiana Periodica 64, 1998, 39-74. 265-303. 


\section{Güvercinlik/Güvercinlik Tepesi}

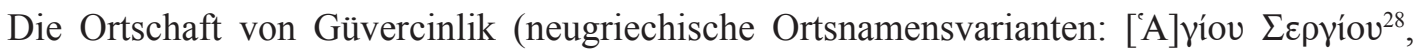

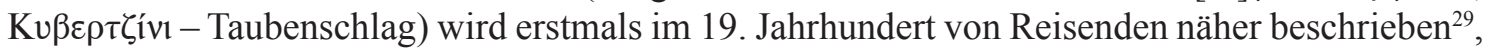
ohne jedoch folgende Beobachtung gemacht zu haben ${ }^{30}$ : Oberhalb des am Ende eines langgezogenen, fjordähnlichen Meeresarmes an einem natürlichen Hafen gelegenen, heutigen türkischen Kleinstädtchens Güvercinlik erhebt sich östlich eine beträchtliche Anhöhe - Güvercinlik Tepesi (Abb. 3). Bei der Begehung stellte sich heraus, daß sich auf der Kuppe dieser Anhöhe

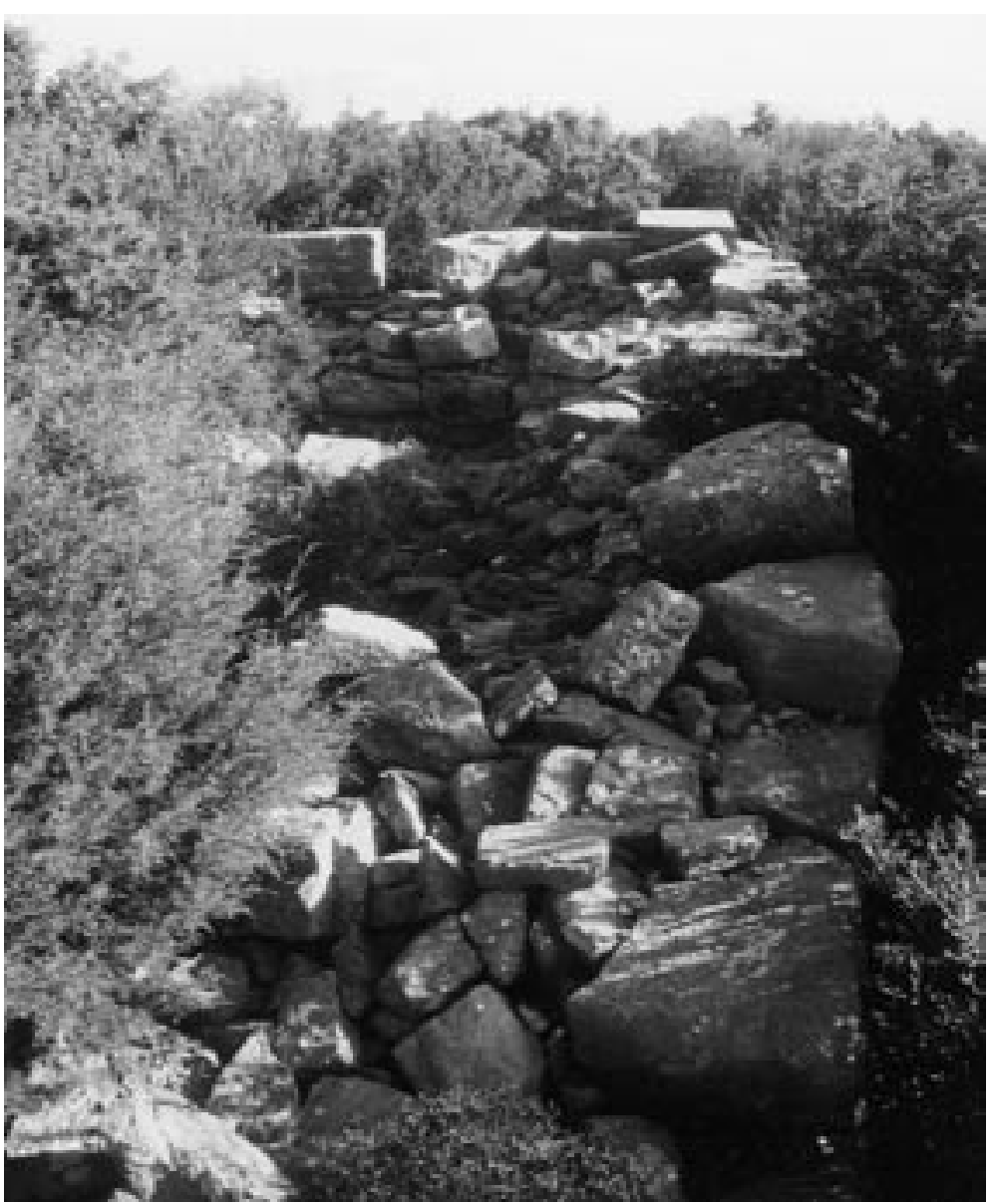
eine antike Siedlung einigen Umfangs befunden haben mußte.

Güvercinlik selbst besitzt heute nur noch geringe Spuren antiker Bebauung und Besiedlung. In den Gärten der Ortschaft und auf den Grundstücken begegnen Ruinen antiker Häuser, Keramik, römische Bauglieder, ferner wurde in der Nähe (westlich in Siralık) ein römisches Inschriftenfragment mit Entfernungsangaben gefunden $^{31}$. Weiterhin legen von Einheimischen verwahrte, vor Ort gefundene spätantike Münzen (iustinianisch bis Kaiser Phokas [602-610]) sowie der Fund eines Fragments byzantinischer Bauplastik (Abb. 4) ${ }^{32}$, das zu einer Schrankenplatte zu ergänzen ist (Abb. 5) ${ }^{33}$, hier ein Weiterbestehen von Ansiedlungen in der frühby-

7 Güvercinlik Tepesi. Ruine des hellenistischen Turms

28 Vgl. Paton - Myres, Karyanda 375: »Karyanda was placed in the Admirality Chart No. 1546 and in Kiepert's last map at Giu Sergiu (Guvernegik: Kiuvergini) the deep bay with ruins and traces of a road south-east of Tarandos Island, but for no adequate reason.« Ebenso belegt ist der Name »Insel Kavergina« für Salih Adas1. Vgl. dazu o. Anm. 11.

29 Newton, Travels II 46: »...[Guverjilik] is a wretched hamlet, once a village, but now consisting about three houses. A turkish custom-house is situated here to levy duties on the pine-timber of the neighbouring forests on its exportation. There is safe anchorage here, but its situation is very unhealthy."

30 Vgl. Newton, History II 600, vgl. o. Anm. 14. Paton - Myres, Karyanda 375, vgl. o. Anm. 28.

31 Ruggieri - Giordano - Zäh, Penisola 123 Abb. 1; Foss, Strobilos 170.

32 Material: feinkristalliner weißer Marmor. Maße: $65,6 \times 39,6 \times 10,1 \mathrm{~cm}$.

33 Auf ein formal entsprechendes Vergleichsbeispiel aus Hierapolis hat mich freundlicher- und dankenswerterweise Herr Dr. Martin Dennert (Freiburg) aufmerksam gemacht. Im Gegensatz zu meinem vierstrahligen Rekonstruktionsvorschlag weist diese - annähernd unversehrt erhaltene - Platte ein zweistrahliges Motiv auf; sie ist allerdings weniger detailliert bearbeitet. G. Ciotta - L. P. Quaglino, La Cattedrale di Hierapolis, in: Hierapolis IV. Scavi e Recherche (2004) 187 Abb 14. Vgl. ebenso: E. Russo in: R. Pillinger u. a. (Hrsg.), Efeso paleocristiana e bizantina 
zantinischen Epoche nahe ${ }^{34}$. In der näheren Binnenumgebung von Güvercinlik (bei Meşelik Köyü) konnte zudem die schlecht erhaltene Ruine einer frühbyzantinischen Basilika festgestellt werden, was die großflächige byzantinische Besiedlung dieser Gegend bestätigt $^{35}$. Die meisten archäologischen Spuren in Güvercinlik drohen allerdings durch die massive Neubautätigkeit schon in Kürze völlig verschwunden zu sein.

Auf der Hügelkuppe von Güvercinlik Tepesi angekommen, bemerkt man zunächst relativ niedrig anstehende weitausgefächerte Mauerstruk-

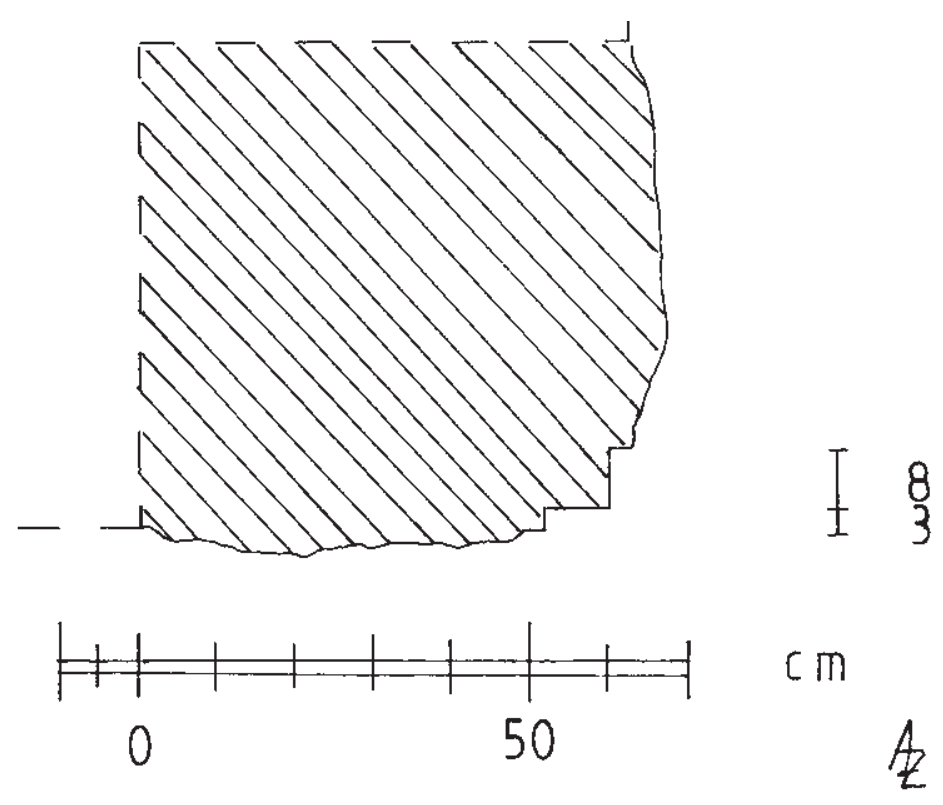

8 Hellenistischer Turm, Eckbaustein mit charakteristischer Eckabarbeitung turen, die - halbkonzentrisch unterhalb der Hügelkuppe angelegt - offenbar zu einer Siedlung gehörten und möglicherweise als Überreste von Wohnbauten zu deuten sind. Der Stil des Mauerwerks, das aus flachen Bruchsteinen zusammengesetzt ist, entspricht den lelegischen Bauten der Halbinsel von Halikarnas$\operatorname{sos}^{36}$. Im Zentrum des Hügels hat der Verfasser die Überreste eines großen, langrechteckigen hellenistischen Turms festgestellt, dessen Ausmaße mit ca. $10 \times 5 \mathrm{~m}$ beträchtlich sind (Abb. 6-7). Er verfügt über die bei fast allen hellenistischen Turmbauten belegten charakteristischen Eckbausteine, sog. Bossenquader mit Randschlag (Abb. 8); dieses Detail läßt sich besonders gut am - seit Herbst 1998 - von einem türkischen Team ausgegrabenen und restaurierten

- Frühchristliches und byzantinisches Ephesos, AForsch 3 (1999) 52 Taf. 30; 74 (iustinianisch). Beherrscht wurde die Ansichtsseite unseres Exemplars von einem stark profilierten konzentrischen Muster mit einer zentralen Kreuzpalmette. Nach außen zeigten ehedem die Spitzen von vier Palmettenblättern, die von Dreiecksprofilen umgeben wurden. Von der Machart ähnlich sind Platten, die einen mehrfach profilierten Rhombus - anstatt der hier belegten Kreise - zeigen. Gemeinsam ist diesen Platten die stark profilierte Gestaltung sowie das im Zentrum der Platten ausgearbeitete kreuzförmige Palmetten-Vierblatt oder eine zentrale konzentrische Blüte. Vgl. dazu Beispiele bei Y. Ötüken, Forschungen im nordwestlichen Kleinasien. Antike und byzantinische Denkmäler in der Provinz Bursa

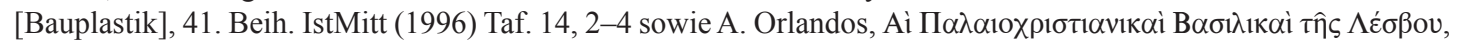
ADelt 12, 1929, 52. 53 Abb. 57. 59. Diese Platten entsprächen auch in der Größe sehr gut unserem rekonstruierten Fragment. Die auffallenden Palmettenblätter waren auch in der frühislamischen Kunst sehr beliebt, vgl. dazu: T. Ulbert, Untersuchungen zu den byzantinischen Reliefplatten des 6. bis 8. Jhs., IstMitt 19/20, 1969/70, 339-357 Abb. 1 (Palmetten) Taf. 71, 2; 73, 2.4 (o. erwähnte Platten mit kompositorischen Parallelen). Das Motiv unserer Platte war auch in frühmittelalterlicher Zeit verbreitet, es kann etwa in Rom (Santa Sabina) nachgewiesen werden. Vgl. M. T. Cecchelli, Corpus della scultura altomedievale VII: La diocesia die Roma IV. La regione ecclesiastica (1976) 215-217 Nr. 246 Taf. 81 (Anfang 9. Jh., mit weiterer Lit. Herzlichen Dank für dieses Zitat wieder an M. Dennert). Historische Abb. bei: J. Strzygowski, Die bildende Kunst des Ostens (1916) Abb. 5.

34 Allerdings schien Karyanda in dieser Zeit keinen Stadtstatus mehr gehabt zu haben, denn es ist weder als Polis noch als Bistum in den Schriftquellen belegt. Es wäre gut möglich, daß Karyanda in byzantinischer Zeit dem nur

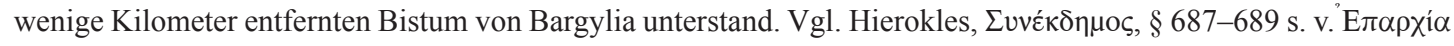
Kapíaৎ (Editionen: A. Burckhardt 1893; E. Honigmann 1939). Eine weitere Möglichkeit bestünde darin, das byzantinische Karyanda für das bisher nicht identifizierte Bistum von »Nea Polis« zu halten. Zu Karien allgemein: RAC XX (2001) 146-166 s. v. Karien (V. Ruggieri).

35 A. Zäh, Zur Typologie kirchlicher Architektur im südwestlichen Kleinasien (2003) 71-72 Abb. 186. Die Ruine befindet sich östlich von Meşelik Köyü.

36 Vgl. Radt, Siedlungen, Taf. 21. 24. 27. 


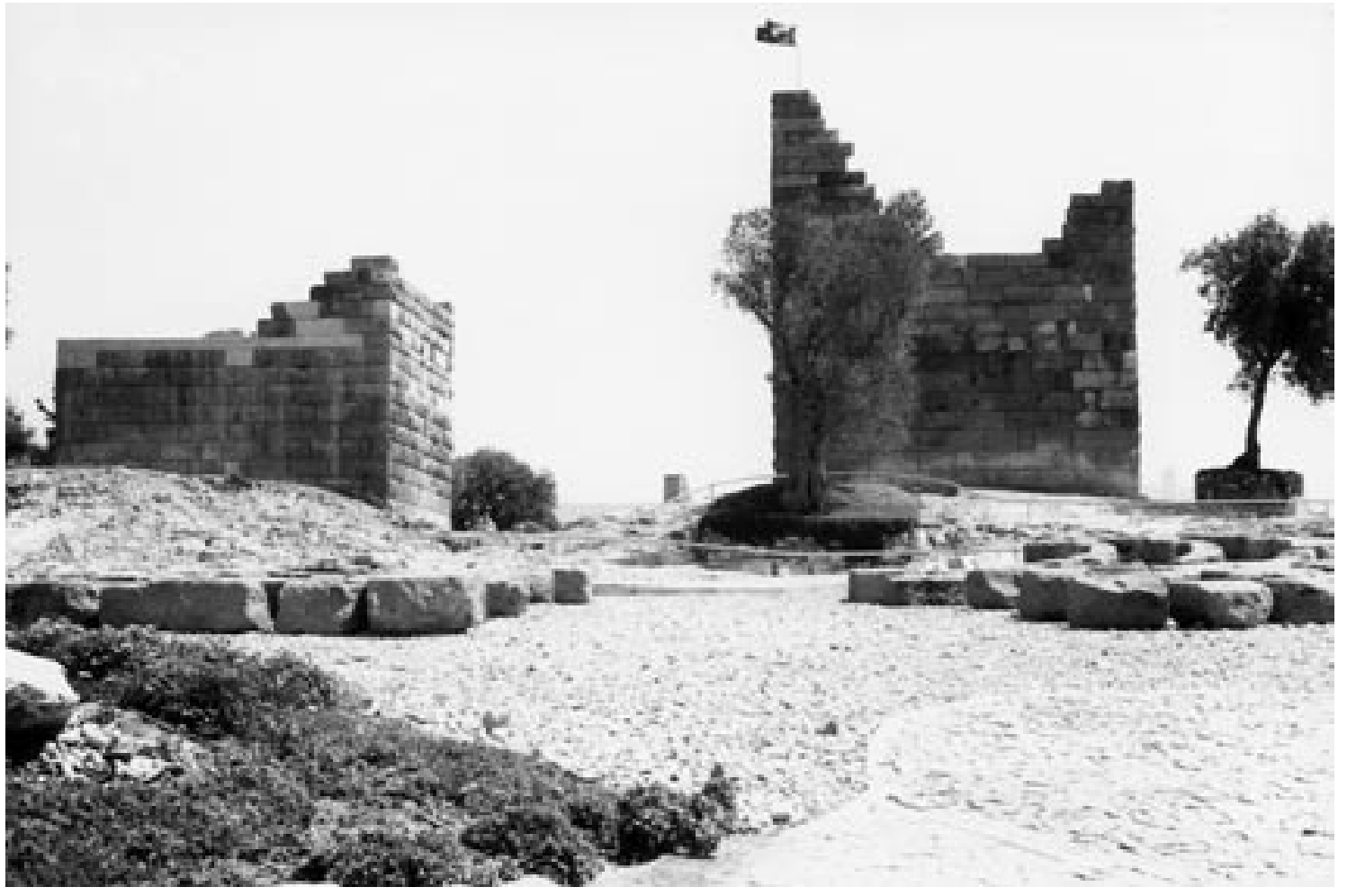

9 Halikarnassos (Bodrum). Myndos-Tor von Westen (aktueller Zustand)

Myndos-Tor der hellenistischen Stadtmauer von Halikarnassos erkennen (Abb. 9) ${ }^{37}$. Im Gegensatz dazu ist das Mauerwerk unseres Turms, das mit 4-5 Hausteinlagen noch mehr als $2 \mathrm{~m}$ hoch über dem Verschüttungshorizont ansteht (vgl. Abb. 6, Nordostecke), allerdings nur pseudoiso$\mathrm{dom}^{38}$. Es war dem Verfasser aufgrund der dichten Vegetation nicht möglich zu überprüfen, ob es sich bei dem Turm um ein isoliertes Einzelbauwerk handelt, oder ob er an einen weiteren Mauerzug im Sinne einer größeren Wacht- oder Wehrstruktur anschloß ${ }^{39}$. Vielleicht ist das Bauwerk als Wacht- oder Signalturm zu deuten ${ }^{40}$, der möglicherweise mit dem Turm von Salih Adas $^{41}$ in Sichtverbindung stand; jedenfalls entsprechen die Abmessungen des Grundrisses in etwa einem der hellenistischen Türme im karischen Alinda ${ }^{42}$. Ferner begegnet dem Besucher hier oben die Ruine eines kleineren Bauwerks mit abgetreppten Sitzstufen (Bouleuterion?), die allerdings völlig verschüttet ist. Besonders beklagenswert sind die auf der Siedlungskuppe überall festzustellenden Spuren extensiver Raubgrabungen, die nahelegen, daß diese - bisher wissenschaftlich unbekannte - Siedlung schon seit längerem planmäßig ausgeplündert wird. Bei meinem Besuch nahm ich größere Bruchstücke korrodierter Kupfer- bzw. Bronzebleche und -gefäße (Abb. 11), die von den Raubgräbern achtlos zurückgelassen worden waren, wahr. Die

${ }_{37}$ McNicoll, Fortifications 16 ff. Vgl. ebenso: Newton, History, Tafelbd.; Newton, Travels II, Taf. 1.

38 Terminologie nach: R. L. Scranton, Greek Walls (1941); vgl. ebenda 74 Abb. 14 (Datierung: 3. Jh. v. Chr.). Vgl. ferner: F. E. Winter, Greek Fortifications (1971) Abb. 162. 192. Ähnlich ebenso eine Mauerecke in Kaunos: B. Schmaltz, Kaunos 1988-1991, AA 1994, 195 ff. Abb. 7. 8 (3. Jh. v. Chr.).

39 McNicoll, Fortifications 171 ff. Eine möglicherweise vergleichbare kleine Befestigungsanlage mit einem hellenistischen Turm befindet sich im nördlichen Latmosgebirge bei Yaylası (Attau-lu-su). McNicoll, Fortifications 41 f.; Paton - Myres, Sites 213 f. Abb. 11 Taf. 9, 2.

40 F. Krischen, Die Befestigungen von Herakleia am Latmos, Milet III 2 (1922) $41 \mathrm{ff}$.

${ }^{41}$ Auf der Insel soll sich ein etwa $10 \mathrm{~m}$ großer - und damit entsprechender - hellenistischer Turm befinden. Bean - Cook, Peninsula 132.

42 McNicoll, Fortifications 26 ff. Abb. 4 Taf. 12. 


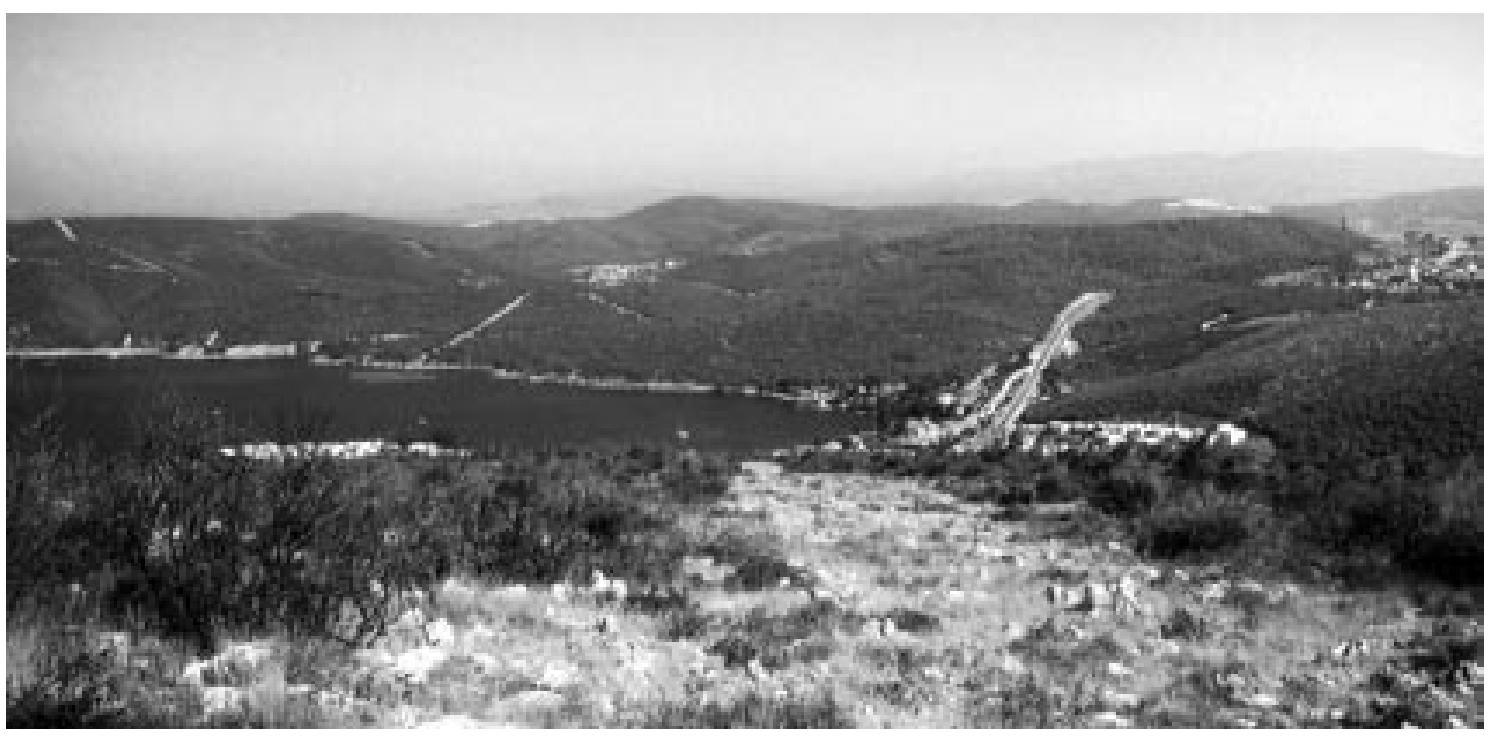

10 Güvercinlik Tepesi. Blick vom Fuß der Kuppe hinab auf Güvercinlik

Oberflächenkeramik entspricht hellenistischen und früheren Gebrauchswaren, wobei auch schwarzglasierte und weißgrundige Keramikfragmente anzutreffen sind.

\section{Zusammenfassung und Ausblick}

Befundtabelle der diskutierten Ortslagen

\begin{tabular}{|l|l|}
\hline \multicolumn{1}{|c|}{ Ortslage } & \multicolumn{1}{c|}{ Bisher festgestellter archäologischer Befund } \\
\hline Yalıkavak Yarımadası & Gräber am Isthmus \\
\hline Gölköyü Hafen & römische Ruinen, byzantinische Ruinen (vom modernen Ort größtenteils überbaut) \\
\hline Gölköyü Tepesi & lelegische Ruinen \\
\hline Tavşan Adası & hellenistische Mauern, byzantinische Klosterkirche \\
\hline Torba & frühbyzantinische Siedlung mit Basilika, Mausoleum, Thermen und Zisterne \\
\hline Salih Adası & lelegische(?) Ruinen, hellenistische Ruinen, byzantinische Ruinen \\
\hline Güvercinlik Köyü & römische Ruinen, byzantinische Ruinen (vom modernen Ort größtenteils überbaut) \\
\hline Güvercinlik Tepesi & lelegische(?) Ruinen, hellenistische Ruinen \\
\hline
\end{tabular}

Schluß

Die in Sichtweite von Güvercinlik gelegene Salih-Insel erscheint aufgrund des positiven archäologischen Befundes - nach wie vor - als idealer Kandidat für die Insel Karyanda. Die Textüberlieferung belegt eine geteilte Identität für Karyanda: eine Insel mit Siedlung und einen Hafen, jedoch nicht einen See mit Siedlung ${ }^{43}$. Die Höhensiedlung von Güvercinlik Tepesi ist möglicherweise als Teil des festländischen, als das am Hafen (Güvercinlik) gelegene Karyanda zu deuten, denn der bedeutende natürliche Hafen von Güvercinlik wurde offenbar während der gesamten Antike genutzt und wäre deshalb auch als der topographisch nächstgelegene und damit lebens-

43 Bean - Cook, Peninsula $156 \mathrm{f}$. 


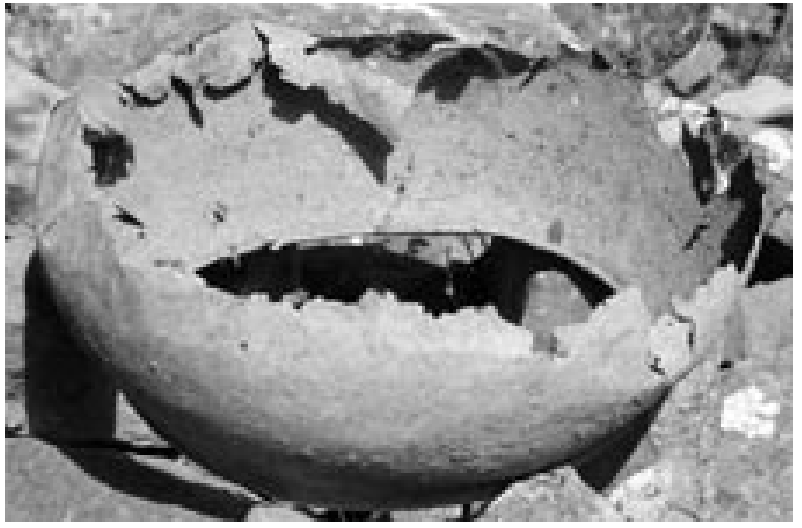

11 Korrodiertes Metallgefäß

wichtige Hafen- und Umschlagplatz für den innerkarischen Binnenhandel der Karyanda-Insel denkbar. Die hellenistische Befestigung der Hügelkuppe deckte den Weg nach der nördlich in unmittelbarer Nachbarschaft gelegenen Polis Bargylia und dem wichtigen karischen Zentrum Mylasa (Milas) ${ }^{44}$ und ermöglichte ebenso den Einblick in die nordöstlich gelegene Kara-Ebene (Kara Ovas1) sowie generell die Überwachung des Küstenabschnitts nach Halikarnassos ${ }^{45}$ (Abb. 10). Besonders unterstützt wird der Nachweis der Siedlung in hellenistischer Zeit auch durch die Tatsache, daß eine Münzprägung von Karyanda bis in das 3. Jahrhundert v. Chr. nachgewiesen ist ${ }^{46}$. Dies korreliert mit den hellenistischen Ruinen auf der Kuppe von Güvercinlik Tepesi. Eine Planaufnahme der in Frage kommenden archäologischen Stätten bei Gölköyü, auf Salih Adası und schließlich Güvercinlik Tepesi im Rahmen eines Surveys wäre ein dringendes Desiderat, um einer Lösungsfindung näher zu kommen.

\section{Dr. Alexander Zäh}

Ascher Straße 45, D-63477 Maintal

E-Mail: alexander.zaeh@t-online.de

Abbildungsnachweis: Abb. 1: nach Amtliche Deutsche Seekarte (1990), Blatt D 613, M 1:150.000, durch Verf. ergänzt und bearbeitet; Abb. 2: nach Bean - Cook (1955) Abb. 14, durch Verf. ergänzt und bearbeitet; Abb. 3-8: Verf.

44 Zum antiken Milas vgl. neben verschiedenen Beiträgen des Verf. zur Stadt u. a.: F. Rumscheid, Mylasas Verteidigung: Burgen statt Stadtmauer?, in: E.-L. Schwandner - K. Rheidt (Hrsg.), Stadt und Umland, DiskAB 7 (1999) 206-222.

45 Schon seit lelegischer Zeit wurden die Bergkuppen dieser Region planmäßig genutzt und dort zahlreiche Wachtund Fluchtburgen errichtet. Vgl. Radt, Siedlungen 13.

${ }^{46}$ Bean - Cook, Peninusla 156 f.: »third century or earlier«. Weitere numismatische Belege bei: RE X (1919) 2246-2247 s. v. Karyanda (Bürchner). 